Bryn Mawr College

Scholarship, Research, and Creative Work at Bryn Mawr

College

Physics Faculty Research and Scholarship

Physics

1994

\title{
Solid State Proton Spin Relaxation and Methyl and t-Butyl Reorientation
}

Peter A. Beckmann

Bryn Mawr College, pbeckman@brynmawr.edu

Hania A. Al-Hallaq

Anne M. Fry

Amy L. Plofker

Brian A. Roe

See next page for additional authors

Let us know how access to this document benefits you.

Follow this and additional works at: http://repository.brynmawr.edu/physics_pubs

Part of the Physics Commons

\section{Custom Citation}

Peter A. Beckmann et al, J. Chem. Phys. 100, 752 (1994).

This paper is posted at Scholarship, Research, and Creative Work at Bryn Mawr College. http://repository.brynmawr.edu/physics_pubs/11

For more information, please contact repository@brynmawr.edu. 
Authors

Peter A. Beckmann, Hania A. Al-Hallaq, Anne M. Fry, Amy L. Plofker, Brian A. Roe, and Jessica A. Weiss 


\title{
Solid state proton spin relaxation and methyl and $\boldsymbol{t}$-butyl reorientation
}

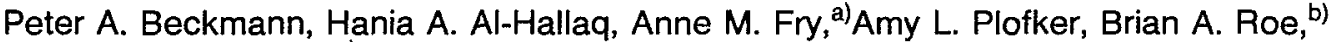 \\ and Jessica A. Weiss ${ }^{\text {c) }}$ \\ Department of Physics, Bryn Mawr College, 101 North Merion Ave., Bryn Mawr, \\ Pennsylvania 19010-2899
}

(Received 9 July 1993; accepted 17 September 1993)

Proton spin relaxation experiments provide a powerful way to study intramolecular reorientation in $t$-butyl substituted benzenes and related systems. A consistent model for the reorientation of $t$-butyl groups and their constituent methyl groups has emerged. ${ }^{1,2}$ These studies have not only provided information on structure and reorientation barriers, but they have also provided insight into the effects that crystal packing has on internal motions.

For a large class of $t$-butyl substituted planar aromatic molecular solids, the local single-molecule symmetry (considering only nearest neighbors on a ring) is one of two types. In one case, there is a plane of symmetry resulting from protons on either side of the $t$-butyl group on the ring. This is the case for the 4-t-butyl group in 1-hydroxy-2,4,6tri-t-butylbenzene (1) and for the 5-t-butyl group in 1-hydroxy-2,5-di-t-butylbenzene (2) as shown in Fig. 1. In this case, the intramolecular electrostatic potential is sixfold and, to within experimental uncertainty, the three methyl groups (called $a$-type methyl groups) and the $t$-butyl group (called an $A$-type $t$-butyl group) are observed to reorient with the same correlation time $\tau_{a}$. In the other case, there is a lower, threefold symmetry where a hydrogen atom is on one side of the $t$-butyl group and another atom or group (often $\mathrm{OH}$ ) is on the other side. This is the case for the 2- and 6-t-butyl groups in 1 and the 2-t-butyl group in 2. In this case, two equivalent methyl groups (called $c$-type methyl groups) are above and below the ring. Their reorientation is characterized by the correlation time $\tau_{c}$. The third methyl group (called a $b$-type methyl group), is in the plane of the ring adjacent to the ring proton. It reorients with a different characteristic correlation time $\tau_{b}$. The $t$-butyl group also reorients with $\tau_{b}$. This has been called a $B$-type $t$-butyl group.

Yamauchi and McDowell (YM) presented a study ${ }^{3}$ using 1. They interpreted their data with the interesting conclusion that $\tau_{a}=\tau_{c}$; that is, the out-of-plane methyl groups in the 2-and 6-t-butyl groups in 1 see the same barrier as the 4-t-butyl group and its constituent methyl groups. We have performed a more thorough Zeeman relaxation experiment with 1 and the data is shown in Fig. 2. The experimental procedures are outlined elsewhere. ${ }^{4} \mathrm{We}$ find a more complicated situation than suggested by YM. YM investigated at only one frequency whereas we have used three (Fig. 2). Also, we have extended the experiments to higher temperatures. YM's results are indicated in Fig. 2 by large equally spaced open circles. These circles adequately represent YM's data and fit. The highest temperature employed by $\mathrm{YM}$ was about $285 \mathrm{~K}$.
The temperature dependence of the relaxation rate for 1 is similar to that for 2 in that there are three sets of overlapping relaxation curves due to the terms involving $\tau_{a}, \tau_{b}$, and $\tau_{c}{ }^{1}$ However, two of the three curves overlap more in 1 (Fig. 2) than in 2 and in order to sort out the motions, experiments are needed at more than one frequency and at sufficiently high temperatures to find the frequency-independent region. The theoretical fit at 53 $\mathbf{M H z}$ is separated into its various constituents in Fig. 2. The theory, the mathematical background and the various models behind the present analysis are presented in detail elsewhere. ${ }^{1,5}$ The single-peaked solid curve labeled $A$ and the double-peaked curve labeled $B$ in Fig. 2 refer to the relaxation resulting from the reorientation of the $A$ - and $B$-type $t$-butyl groups and their constituent methyl groups. Curve $A$ results from the superimposed reorientation of the $A$-type $t$-butyl group and its three $a$-type methyl groups. The three dashed curves that make up the $B$ term are labeled $B b, c$ and $B c$. Curve $B b$ results from the superposition of the reorientation of the $B$-type $t$-butyl groups and their single $b$-type (in-plane) methyl groups. Curve $c$ results from the reorientation of the $c$-type (out-of-plane) methyl groups in the $B$-type $t$-butyl groups and curve $B c$ results from the superposition of this faster $c$-type reorientation and the slower reorientation of the $B$-type $t$-butyl group. This $B c$ term involves both $\tau_{b}$ and $\tau_{c}$.

The temperature dependence of the observed relaxation rate in 1 has two unusual and interesting properties. First, the uncertainties in the observed rates, about $5 \%$, are much less than the spread in the data points indicated in Fig. 2. This results from the various thermal histories of the solid. We performed many thermal histories and one reason there are so many data points in Fig. 2 is that we made measurements until we established the range of values. Any given day resulted in a smooth set of data with
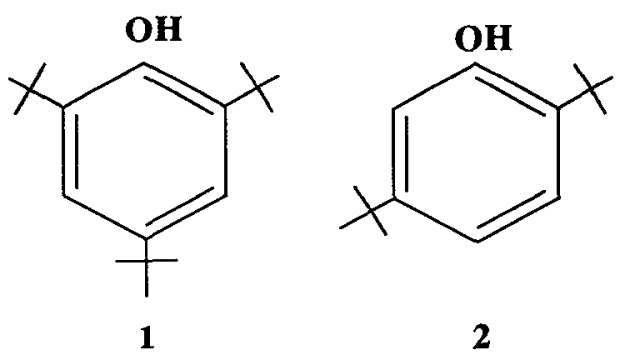

FIG. 1. Schematic drawings of (1) 1-hydroxy-2,4,6-tri-t-butylbenzene, and (2) 1-hydroxy-2,5-di-t-butylbenzene. 
very little scatter in neighboring data points, much like the data presented by YM. The sample of $\mathbf{1}$ was recrystallized, pumped and sealed but we note that earlier exploratory experiments on a "dirtier" unsealed sample resulted in a much greater spread of data points.

The other interesting feature found from analyzing the data in Fig. 2 is that there is a significant distribution of barriers that characterize $\tau_{b}$. Looking at $\tau_{a}$ and $\tau_{c}$ first, the relaxation rates indicated by curves $A$ and $c$ in Fig. 2 are each given by an appropriate sum of unique- $\tau$ spectral densities $\tau_{i}\left(1+n^{2} \omega^{2} \tau_{i}^{2}\right)^{-1}$ for $i=a$ or $c, n=1$ and 2, Larmor frequency $\omega / 2 \pi$ and correlation time $\tau_{i}=\tau_{i \infty} \exp \left(E_{i} /\right.$ $k T){ }^{1,5}$ The barriers $E_{a}=6.7 \mathrm{~kJ} \mathrm{~mole}^{-1}$ and $E_{c}=10 \mathrm{~kJ}$ $\mathrm{mol}^{-1}$ for 1 can be compared with the values $E_{a}=6.2$ $\mathrm{kJ}$ mole ${ }^{-1}$ and $E_{c}=15 \mathrm{~kJ}$ mole ${ }^{-1}$ for 2 . The uncertainties in these barriers are large; about $\pm 25 \%$, but they tell a consistent story nonetheless. Whereas the $c+B c$ and $A$ curves coalesce in 1 at lower temperatures (Fig. 2), they are well separated in 2 . $^{1} \mathrm{YM}$ give $E_{a}=E_{c}=3.8 \mathrm{~kJ}$ mole ${ }^{-1}$ for 1 and they comment on the fact that this value is unusually small. ${ }^{3}$ They arrive at this small value because they have not appropriately divided the broad low-temperature relaxation curve into its constituent parts. The relaxation resulting from the superposition of the reorientation of the 2 - and 6-t-butyl groups ( $B$-types) and their constituent in-plane methyl groups ( $b$-types) in $\mathbf{1}$ is characterized by a broad range of barriers. This manifests itself in the fact that the low-temperature slope of the $B b$ curve is much smaller than the high-temperature slope (whereas the $A, B c$, and $c$ curves have equal high- and low-temperature slopes). The ratio of the slopes for curve $B b$ is 0.3 ; this is referred to as the distribution parameter $\epsilon$ in the theories. ${ }^{1,5,6}$ There is no way around fitting this with a large distribution of $\tau_{b}$ values. No finite sum of functions of the form $\tau\left(1+\omega^{2} \tau^{2}\right)^{-1}$ will produce the observed result. In producing curve $B b$, we have used a Davidson-Cole distribution ${ }^{6}$ of correlation times $\tau_{b}$ but a Frolich distribution ${ }^{6}$ would work just as well. Both distributions were used for $2{ }^{1}$ The upper cutoff value $E_{b}$ (which, along with $\epsilon$, characterizes the distribution of correlation times or barriers) is $20 \mathrm{~kJ} \mathrm{~mole}^{-1}$ for 1 and this can be compared with $28 \mathrm{~kJ} \mathrm{~mole}^{-1}$ for $2 .^{1} \mathrm{YM}$ arrived at the much lower value of $13 \mathrm{~kJ} \mathrm{~mole}^{-1}$ but this is because they did not go to sufficiently high temperature and they fitted data arrived at by incorrectly subtracting off the low temperature data. The $B$ curve cannot easily be separated since the $B c$ component involves both $\tau_{b}$ and $\tau_{c}$.

In summary, the reorientation of the $t$-butyl groups and their constituent methyl groups in a large class of $t$-butyl-substituted benzenes can be interpreted by a simple,

\section{$\mathbf{T}$ (K)}

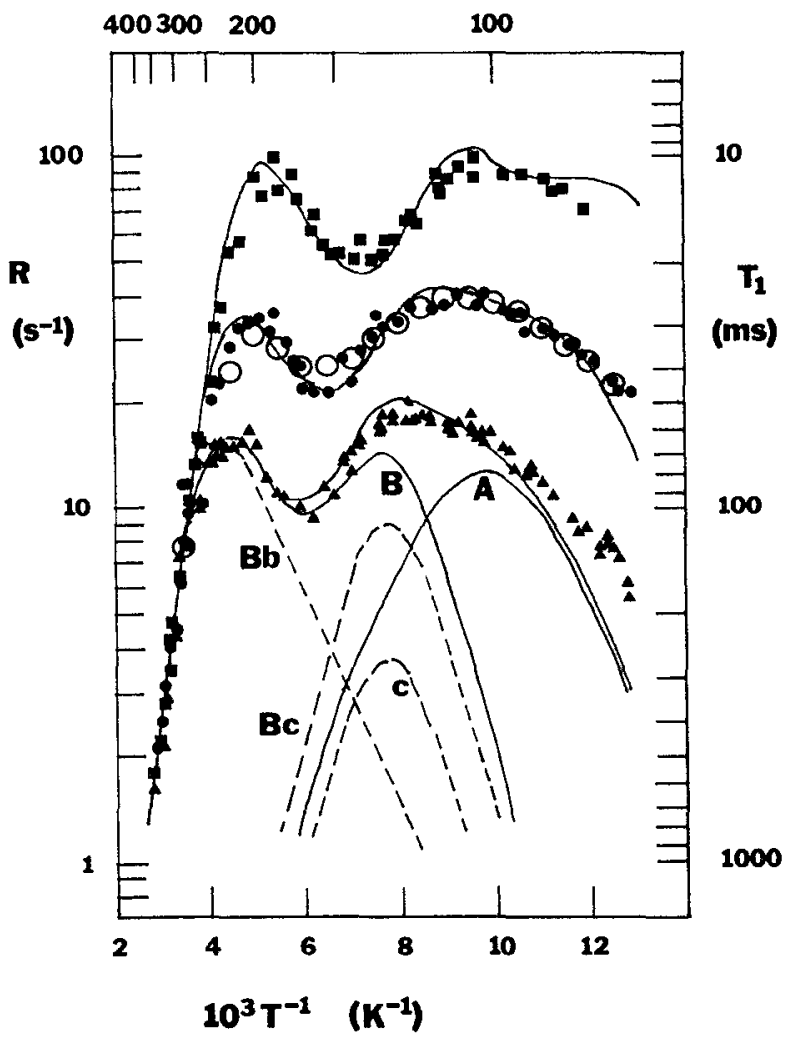

FIG. 2. The temperature $T$ dependence of the proton Zeeman relaxation rates $R$ at Larmor frequencies of $8.50(\mathbf{\omega}), 22.5(\bullet)$ and $53.0 \mathrm{MHz}(\Delta)$ in polycrystalline 1-hydroxy-2,4,6,-tri-t-butylbenzene (1). The data and the theoretical fit of Yamauchi and McDowell (Ref. 3) is represented by the equally spaced $O$ 's. The lines are discussed in the text.

quite general model. In arriving at parameters that characterize the reorientation process, reasonably extensive experiments must be carried out.

a) Department of Physics and Astronomy, CB\# 3255, Phillips Hall, University of North Carolina, Chapel Hill, NC 27599-3255.

${ }^{b}$ Graduate Field of Environmental Toxicology, 215 Rice Hall, Cornell University, Ithica, NY 14853.

${ }^{c}$ Department of Chemistry, James-Franck Institute, University of Chicago, 5640 S. Ellis Avenue, Chicago, IL 60637.

${ }^{1}$ P. A. Beckmann, A. M. Cheung, E. E. Fisch, F. A. Fusco, R. E. Herzog, and M. Narasimhan, J. Chem. Phys. 84, 1959 (1986).

${ }^{2}$ P. A. Beckmann, Phys. Rev. B 39, 12248 (1989).

${ }^{3}$ J. Yamauchi and C. A. McDowell, J. Chem. Phys. 75, 1051 (1981).

${ }^{4}$ P. A. Beckmann, A. I. Hill, E. B. Kohler, and H. Yu, Phys. Rev. B 38, 11098 (1988).

${ }^{5}$ P. A. Beckmann, R. M. Hathorn, and F. B. Mallory, Mol. Phys. 69, 411 (1990).

${ }^{6}$ P. A. Beckmann, Phys. Rep. 171, 85 (1988). 